\title{
THE SYNTACTIC MONOID OF AN INFIX CODE
}

\author{
MARIO PETRICH AND GABRIEL THIERRIN
}

(Communicated by Warren J. Wong)

\begin{abstract}
Necessary and sufficient conditions on a monoid $M$ are found in order that $M$ be isomorphic to the syntactic monoid of a language $L$ over an alphabet $X$ having one of the following properties. In the first theorem $L$ is a $P_{L}$-class and $P_{W(L)} \subseteq P_{L}$ where $P_{L}$ is the syntactic congruence of $L$ and $W(L)$ is the residue of $L$. In the second theorem $L$ is an infix code; that is, satisfies $u, u v w \in L$ implying $u=w=1$. In the third theorem $L$ is an infix code satisfying a condition which amounts to the requirement that $M$ be a nilmonoid. Various refinements of these conditions are also considered.
\end{abstract}

\section{INTRODUCTION AND SUMMARY}

For any set $X$, a language $L$ is any subset of $X^{*}$, the free monoid on $X$. In the study of properties and classification of languages, $L$ is usually specialized to satisfy some strong conditions that make it amenable to various kinds of treatments. One of the most common restrictions is the requirement that $L$ be a code; that is, a set of free generators for the submonoid of $X^{*}$ generated by $L$. Further specialization leads to the rich family of codes, among which the infix codes figure prominently. A language $L$ is an infix code if it is an antichain for the partial order on $X^{*}$ called the infix order.

Going back to general languages, one of the most successful tools of studying them is the syntactic monoid. For a language $L$ over $X$, one first constructs the syntactic congruence $P_{L}$ (the greatest congruence on $X^{*}$ saturating $L$ ) and the quotient monoid, $\operatorname{Syn}(L)=X^{*} / P_{L}$, is the syntactic monoid of the language $L$. Monoids isomorphic to syntactic monoids of languages are easily characterized as monoids containing a disjunctive subset $D$ (that is, the obvious analogue of the syntactic congruence for $D$ is the equality relation). In various special cases, deeper relations between the properties of the language and the properties of its syntactic monoid can be discovered.

Received by the editors May 5, 1989.

1980 Mathematics Subject Classification (1985 Revision). Primary 20M05, 20M35; Secondary $68 \mathrm{~A} 30$

Key words and phrases. Monoid, language, congruence, syntactic, code, infix.

This research was supported by the Natural Sciences and Engineering Research Council of Canada, Grant A7877. 
It should now be clear that if we combine the subjects of the preceding two paragraphs, we are naturally led to a study of the syntactic monoid of an infix code. As should be expected, such a syntactic monoid will have some strong properties. In the first place, since an infix code $L$ always has a nonempty residue $W(L)$, which is then a $P_{L}$-class, we get that $\operatorname{Syn}(L)$ has a zero. Furthermore, since $L$ itself is a $P_{L}$-class we know that $\operatorname{Syn}(L)$ has a disjunctive element. Finally, since $P_{L}=P_{W(L)}$, it follows that the zero is a disjunctive element of $\operatorname{Syn}(L)$. Continuing in this vein, we get necessary and sufficient conditions on a monoid $M$ to be isomorphic to the syntactic monoid of an infix code. Further specializations of either the infix code $L$ directly or of its syntactic monoid $\operatorname{Syn}(L)$ are of particular interest. For precedences of this study, see [2, 4].

Section 2 contains necessary preliminaries. A characterization of $\operatorname{Syn}(L)$ when $L$ is a $P_{L}$-class and $P_{W(L)} \subseteq P_{L}$ is the essence of $\S 3$. Several characterizations of $\operatorname{Syn}(L)$ when $L$ is an infix code are given in $\S 4$. Finally, several consequences of this result are treated in $\S 5$.

\section{Preliminaries}

A language $L$ over an alphabet $X$ is any subset of $X^{*}$, the free monoid on $X$ consisting of words over $X$ with juxtaposition as product. The syntactic congruence $P_{L}$ of $L$ defined on $X^{*}$ is

$$
u \equiv v \quad\left(P_{L}\right) \text { if } x u y \in L \Leftrightarrow x v y \in L \text { for all } x, y \in X^{*} .
$$

The quotient monoid $\operatorname{Syn}(L)=X^{*} / P_{L}$ is the syntactic monoid of $L$. Further $\bar{L}$ denotes the complement of $L$ in $X^{*}$. The identity of $X^{*}$ is denoted by 1 ; the symbol $\varnothing$ stands for the empty set except when it is the identity of $X^{*}$. The complement of 1 in $X^{*}$ is the free semigroup $X^{+}$on $X$. For $w \in X^{*}$, $w^{*}=\left\{w^{n} \mid n \geq 1\right\} \cup\{1\}$. A language $L$ over $X$ is an infix code if it is nonempty, does not contain 1 , and for any $u, v, w \in X^{*}, v, u v w \in L$ implies $u=$ $w=1$. Hence it can be characterized as an antichain in $X^{+}$under the infix order. For $u, v \in X^{*}, u \leq v$ in the embedding order if $u=x_{1} x_{2} \ldots x_{n}$, $v=y_{1} x_{1} y_{2} x_{2} \ldots x_{n} y_{n+1}$ for some $x_{i}, y_{i} \in X^{*}$. A nonempty antichain, not containing 1 , in this order is a hypercode. A hypercode is obviously an infix code. It is well known that if $L$ is a hypercode over a finite alphabet $X$, then $L$ is itself finite.

The above definition of $P_{L}$ is used for any subset $L$ of any semigroup $S$. If $L=\{a\}$, we write $P_{a}$ instead of $P_{\{a\}}$. If $P_{L}$ is the equality relation, $L$ is a disjunctive subset of $S$; if $L=\{a\}$, we say that $a$ is a disjunctive element of $S$. For any $a \in S, J(a)$ denotes the principal ideal of $S$ generated by $a$. If $S$ has a zero, the intersection of all nonzero ideals, if different form zero, is the core of $S$, denoted by core $(S)$; the set

$$
\mathscr{A}(S)=\{a \in S \mid a s=s a=0 \text { for all } s \in S\}
$$

is the annihilator of $S$. A trivial semigroup has a core by definition. 
For any sets $A$ and $B, A \backslash B=\{a \in A \mid a \notin B\}$. If $\varphi$ is a mapping defined on $A$, then $\bar{\varphi}$ denotes the equivalence relation on $A$ induced by $\varphi$.

\section{THE FIRST THEOREM}

The first theorem provides necessary and sufficient conditions on a monoid to be isomorphic to the syntactic monoid of a language $L$ such that $L$ is a $P_{L}$-class and $P_{W(L)} \subseteq P_{L}$. The following lemma is well known.

Lemma 1. Let $M$ be a monoid. Let $X=M$ and define a mapping $\varphi$ on $X^{*}$ :

$$
\varphi: x_{1} x_{2} \ldots x_{n} \longrightarrow x_{1} x_{2} \ldots x_{n} \in M
$$

Then $\varphi$ is a homomorphism of $X^{*}$ onto $M$.

Theorem 1. A monoid $M$ is isomorphic to the syntactic monoid of a language $L$ such that $L$ is a $P_{L}$-class and $P_{W(L)} \subseteq P_{L}$ if and only if $M$ has a disjunctive zero and a core.

Proof.

Necessity. First assume that $W(L)=\varnothing$. Then $P_{W(L)}$ is the universal relation and the hypothesis implies also that $P_{L}$ is the universal relation. But then $L=X^{*}$ and $M$ is the trivial monoid that evidently satisfies the desired conclusion.

Suppose next that $W(L) \neq \varnothing$. Then $W(L)$ is a $P_{L}$-class yielding $P_{L} \subseteq P_{W(L)}$ that together with the hypothesis gives $P_{W(L)}=P_{L}$. In view of the fact that $W(L)$ is the zero of $\operatorname{Syn}(L)$, we conclude that the zero of $M$ is disjunctive. Since $L$ is a $P_{L}$-class, we have $W(L) \neq X^{*}$. Let $v \in X^{*} \backslash W(L)$. Then $X^{*} v X^{*} \cap L \neq \varnothing$ so that $u v w \in L$ for some $u, w \in X^{*}$. Letting $c$ be the image of $L$ in $\operatorname{Syn}(L)$ and for any $x \in X^{*}$, and [x] the image of $x$ in $\operatorname{Syn}(L)$, we obtain $c=[u][v][w]$. This means that $c$ is contained in the ideal of $\operatorname{Syn}(L)$ generated by [v]. Therefore $c$ is in the core of $\operatorname{Syn}(L)$, and since $\operatorname{Syn}(L)$ has a core, so does $M$.

Sufficiency. Let $X=M$. If $M$ is trivial, we may let $L=X^{*}$ and all the required conditions are satisfied. By Lemma 1, we have a homomorphism $\varphi$ of $X^{*}$ onto $M$. Let $c$ be a nonzero element of the core of $M$ and let $L=c \varphi^{-1}$ and $W=0 \varphi^{-1}$. By hypothesis, 0 is disjunctive which easily gives that $P_{W}=\bar{\varphi}$.

Let $u, v \in X^{*}$ be such that $u \varphi=b \varphi$. Assume that $x u y \in L$ for some $x, y \in X^{*}$. Then $(x \varphi)(u \varphi)(y \varphi)=c$ and hence $(x \varphi)(v \varphi)(y \varphi)=c$ giving $x v y \in L$. By symmetry, we conclude that $u \equiv v \quad\left(P_{L}\right)$. Therefore $P_{W}=\bar{\varphi} \subseteq$ $P_{L}$, as required.

We show next that $W=W(L)$. If $w \in W$, then $w \varphi=0$ and hence $(u \varphi)(w \varphi)(v \varphi) \neq c$ for all $u, v \in X^{*}$ and thus $X^{*} w X^{*} \cap L=\varnothing$; that is, $w \in W(L)$. Conversely, let $w \in W(L)$. Then $X^{*} w X^{*} \cap L=\varnothing$ and thus $c \notin J(w \varphi)$. Since $c$ is contained in all nonzero ideals of $M$, we must have 
$J(w \varphi)=0$. Therefore $w \varphi=0$ so that $w \in W$. Consequently $W=W(L) \neq$ $\varnothing$ and hence $W$ is a $P_{L}$-class which implies that $P_{L} \subseteq P_{W}$. In conclusion, $P_{L}=P_{W}=\bar{\varphi}$, and since $L=c \varphi^{-1}$, it follows that $L$ is a $P_{L}$-class, as required.

Remarks. The condition that $L$ is a $P_{L}$-class is equivalent to

$$
u, v \in X^{*}, \quad u L v \cap L \neq \varnothing \Rightarrow u L v \subseteq L,
$$

see [1, Theorem 10.6]. The condition $P_{W(L)} \subseteq P_{L}$ is equivalent to $P_{L}=P_{W(L)}$ since if $W(L)=\varnothing$, then $P_{W(L)}$ is the universal relation, hence $P_{L} \subseteq P_{W(L)}$; in addition, if $W(L) \neq \varnothing$, then it is a $P_{L}$-class, and again $P_{L} \subseteq P_{W(L)}$.

Example 1 below shows that a language $L$ can be a $P_{L}$-class but $P_{W(L)} \nsubseteq P_{L}$. In the next section Example 2 will exhibit a language $L$ that is not a $P_{L}$-class but $P_{W(L)} \subseteq P_{L}$. The two conditions therefore are independent. These examples will also show that the conditions of 0 being disjunctive and the existence of a core are independent.

Example 1. Let $L=\left\{a^{m} b a^{n} \mid m, n \geq 1\right\}$ with $X=\{a, b\}$. For any $m, n \geq 1$ and $u, v \in X^{*}$, we have $u a^{m} b a^{n} v \in L$ if and only if $u, v \in a^{*}$, implying that $L$ is a $P_{L}$-class. Also

$$
\text { uabav } \notin W(L) \Leftrightarrow u, v \in a^{*} \Leftrightarrow u b v \notin W(L)
$$

which implies that $a b a \equiv b \quad\left(P_{(W(L)}\right)$. Since $a b a \not \equiv b \quad\left(P_{L}\right)$, it follows that $P_{W(L)} \nsubseteq P_{L}$.

In the proof of necessity of Theorem 1, we see that $L$ being a $P_{L}$-class alone implies that $\operatorname{Syn}(L)$ has a core. Hence this is true in our example as well. If 0 was disjunctive in this example, Theorem 1 would imply that $P_{W(L)} \subseteq P_{L}$, which is not the case. Therefore 0 is not disjunctive for this $L$.

\section{THE SECOND THEOREM}

It contains three characterizations of the syntactic monoid of an infix code and represents the main result of the paper.

Lemma 2. Let $L$ be a language over an alphabet $X$. If one of the $P_{L}$-classes is an infix code, then the $P_{L}$-class of 1 is trivial.

Proof. Let $C$ be a $P_{L}$-class, an infix code, and let $u \equiv 1\left(P_{L}\right)$. For any $v \in C$, we then get $u v \equiv v \quad\left(P_{L}\right)$. But then $v, u v \in C$ and hence $u=1$ since $C$ is infix. Thus the $P_{L}$-class of 1 is trivial.

Lemma 3. Let $M$ be a monoid with identity $e$ and assume that $M \backslash\{e\}$ is a subsemigroup of $M$. Let $X=M \backslash\{e\}$ and define a mapping on $X^{*}$ :

$$
\varphi: x_{1} x_{2} \ldots x_{n} \quad \longrightarrow \quad x_{1} x_{2} \ldots x_{n} \in M \backslash\{e\}
$$

if $x_{1}, x_{2}, \ldots, x_{n} \in M \backslash\{e\}$ and

$$
\varphi: 1 \longrightarrow e
$$


Then $\varphi$ is a homomorphism of $X^{*}$ onto $M$ such that $w \varphi=e$ if and only if $w=1$. If $c$ is a disjunctive element of $M$, then for $L=c \varphi^{-1}$, we have $\bar{\varphi}=P_{L}$.

Proof. Standard.

Theorem 2. The following conditions on a monoid $M$ with identity $e$ are equivalent.

(i) $M$ is isomorphic to the syntactic monoid of an infix code $L$.

(ii) $(\alpha) M \backslash\{e\}$ is a subsemigroup of $M$;

( $\beta) M$ has a zero;

$(\gamma) M$ has a disjunctive element $c$ such that $c \notin\{e, 0\}$ and $c=x c y$ implies $x=y=e$.

(iii) $(\alpha)$;

( $\delta) \quad M$ has a disjunctive zero;

$(\epsilon) \operatorname{core}(M)=\{c, 0\}$ with $c \in \mathscr{A}(M \backslash\{e\})$.

(iv) $(\alpha),(\delta)$;

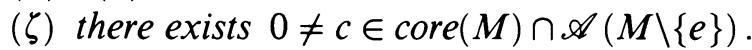

Proof. (i) $\Rightarrow$ (ii). Since $L$ is an infix code, [2, Proposition 1] gives that $L$ is $P_{L}$-class and hence Lemma 2 yields that the $P_{L}$-class of 1 is trivial. Hence $(\alpha)$ holds for $\operatorname{Syn}(L)$ and thus also for $M$. If $u \in L$ and $v \in X^{+}$, then $X^{*} u v X^{*} \cap L=\varnothing$ since $L$ is infix and hence $u v \in W(L)$. Thus $\operatorname{Syn}(L)$ has a zero and therefore $(\beta)$ holds. Let $c$ be the image of $L$ in $\operatorname{Syn}(L)$. Since $L \subseteq X^{+}$by hypothesis, we have $c \neq e$, and since $L \cap W(L)=\varnothing$, we also have $c \neq 0$. It follows easily that $c$ is disjunctive. For any $w \in X^{*}$, let $[w]$ be its image in $\operatorname{Syn}(L)$, and let $u \in L$. Assume that for $x, y \in X^{*}$ we have $c=[x] c[y]$. Then $u, x u y \in L$ implies that $x=y=1$ whence $[x]=[y]=e$. Therefore $M$ satisfies $(\gamma)$ as well.

(ii) $\Rightarrow$ (iii). Let $a \in M \backslash\{0\}$. Since $c$ is disjunctive, there exist $x, y \in M$ such that either $x a y=c$ and $x 0 y \neq c$ or $x a y \neq c$ and $x 0 y=c$. Since the second alternative is impossible, we have $x a y=c$. It follows that $c$ is contained in every nonzero ideal of $M$ so that $c \in \operatorname{core}(M)$.

Since $P_{c}$ is the equality relation, we have $P_{c} \subseteq P_{0}$. For the opposite inclusion, it suffices to show that $\{c\}$ is a $P_{0}$-class. Let $d \equiv c\left(P_{0}\right)$. Then $d \neq 0$ since 0 is a $P_{0}$-class. As above, there exist $x, y \in M$ such that $c=x d y$. In addition, $x d y \equiv x c y \quad\left(P_{0}\right)$ and hence $c \equiv x c y \quad\left(P_{0}\right)$. It follows that $x c y \neq 0$ so that, as above, there exist $u, v \in M$ such that $u x c y v=c$. Then $(\gamma)$ gives $u x=y v=e$ which by $(\alpha)$ yields $x=y=e$ so that $c=d$. Therefore $P_{0} \subseteq P_{c}$ and equality prevails. Therefore 0 is disjunctive and $(\delta)$ holds.

Next let $x \in M \backslash\{0\}$. If $x c \neq 0$, then $c=u x c v$ for some $u, v \in M$, as above. But then $(\gamma)$ and $(\alpha)$ imply that $x=e$. Similarly $c x \neq 0$ implies that $x=e$. It follows that $x c=c x=0$ for all $x \in M \backslash\{e\}$ and, therefore, $c \in \mathscr{A}(M \backslash\{e\})$. 
As a consequence, we have that $I=\{c, 0\}$ is a nonzero ideal of $M$ whence core $(M) \subseteq I$. We have seen above that $c \in \operatorname{core}(M)$ so that $I \subseteq \operatorname{core}(M)$ and equality prevails. Consequently $(\epsilon)$ holds.

(iii) $\Rightarrow$ (iv) trivially.

(iv) $\Rightarrow$ (i). We adopt the result and the notation of Lemma 3 so that $\operatorname{Syn}(L) \cong M$. It remains to show that $L$ is infix. Let $v, u v w \in L$. Then $v \varphi=(u \varphi)(v \varphi)(w \varphi)$ and we have $c=(u \varphi) c(v \varphi)$. It follows that $(u \varphi) c \neq 0$ and $c(v \varphi) \neq 0$ which by $(\zeta)$ yields $u \varphi=v \varphi=e$. By Lemma 3, we conclude that $u=v=1$ so that $L$ is indeed an infix code.

Remarks. In the presence of condition $(\delta)$, according to [3, Theorem 3.7], condition $(\epsilon)$ is equivalent to $M$ being subdirectly irreducible. Even though this latter notion is a more familiar one, the existence of a core is a condition easier to verify.

The following example shows that in condition $(\gamma)$ the implication $c=$ $x c y \Rightarrow x=y=e$ can not be omitted.

Example 2. Let $L=\left\{a^{m} b^{n} \mid m, n \geq 0\right\}$ with $X=\{a, b\}$. Then $P_{L}$ has the following nonresidue classes:

$$
e=\{1\}, f=a^{*}, g=b^{*}, c=\left\{a^{m} b^{n} \mid m, n \geq 1\right\}
$$

and $\operatorname{Syn}(L)$ is isomorphic to the monoid $M=\{e, f, g, c, 0\}$ with the following multiplication table.

\begin{tabular}{l|lllll}
\multicolumn{1}{c}{} & \multicolumn{1}{c}{$\boldsymbol{e}$} & $f$ & $g$ & $c$ & 0 \\
\cline { 2 - 6 } $\boldsymbol{e}$ & $\boldsymbol{e}$ & $f$ & $g$ & $c$ & 0 \\
$f$ & $f$ & $f$ & $c$ & $c$ & 0 \\
$g$ & $g$ & 0 & $g$ & 0 & 0 \\
$c$ & $c$ & 0 & $c$ & 0 & 0 \\
0 & 0 & 0 & 0 & 0 & 0
\end{tabular}

Simple verification shows that $c$ is a disjunctive element of $M$. Here $c=c g$ with $g \neq 1$. Therefore $M$ satisfies conditions $(\alpha),(\beta)$, and $(\gamma)$, except for the implication $c=x c y \Rightarrow x=y=e$, in Theorem 2. The last condition should also be added to [2, Corollary 7]. Clearly $L$ is not an infix code.

In this example we also have that $L$ is not a $P_{L}$-class but, since $W(L)=\bar{L}$, we have $P_{W(L)}=P_{L}$. Since $c$ is a disjunctive element of $M$, this shows that 0 is a disjunctive element too. In addition, $M$ has no core. This example was promised before Example 1 in connection with Theorem 1.

Lemma 4. Let $L$ be a hypercode over an alphabet $X$. Then every nonresidue $P_{L}$-class is a hypercode.

Proof. Let $u, v \in X^{*}$ be such that $u \leq v$ in the embedding order, $u \equiv v\left(P_{L}\right)$ and $u \notin W(L)$. Hence $X^{*} u W^{*} \neq \varnothing$ so that there exist $s, t \in X^{*}$ such that $s u t \in L$. Since $s u t \equiv s v t \quad\left(P_{L}\right)$, it follows that $s v t \in L$. Now $s u t \leq s v t$ implies that $s u t=s v t$ since $L$ is a hypercode and thus $u=v$. 
Lemma 4 does not hold if we substitute hypercode by infix. For example, let $L=\left\{a b^{n} a \mid n \geq 1\right\}$. Then $L$ is infix but the $P_{L}$-class $\left\{b^{n} \mid n \geq 1\right\}$ is not.

Corollary 1. The following conditions on a monoid $M$ with identity $e$ are equivalent.

(i) $M$ is isomorphic to the syntactic monoid of a hypercode.

(ii) $(\alpha)$ and $\beta$ from Theorem 2;

$\left(\gamma^{\prime}\right) M$ has a disjunctive element $c$ such that $c \notin\{e, 0\}$;

$\left(\delta^{\prime}\right)$ if $a_{1} a_{2} \ldots a_{n}=x_{1} a_{1} x_{2} a_{2} \ldots x_{n} a_{n} x_{n+1} \neq 0$, then $x_{1}=x_{2}=\cdots=$ $x_{n+1}=e$.

(iii) $(\alpha)$ and $\beta$ from Theorem 2;

$\left(\gamma^{\prime \prime}\right) M$ has a disjunctive element $c$ such that $c \notin\{e, 0\}$ and if $c=$ $a_{1} a_{2} \ldots a_{n}=x_{1} a_{1} x_{2} a_{2} \ldots x_{n} a_{n} x_{n+1}$, then $x_{1}=x_{2}=\cdots=x_{n+1}=e$.

Proof. (i) $\Rightarrow$ (ii). Since a hypercode is an infix code, conditions $\alpha, \beta$, and $\gamma$ of Theorem 2 are valid here. Assume the antecedent of $\left(\delta^{\prime}\right)$ in $\operatorname{Syn}(L)$. Let $u_{i}, v_{i} \in X^{*}$ be such that $\left[u_{i}\right]=a_{i}$ and $\left[v_{i}\right]=x_{i}$ for all relevant indices $i$. Then

$$
u_{1} u_{2} \ldots u_{n} \equiv v_{1} u_{1} v_{2} u_{2} \ldots v_{n} u_{n} v_{n+1}\left(P_{L}\right)
$$

and $u_{1} u_{2} \ldots u_{n} \notin W(L)$. Now $u_{1} u_{2} \ldots u_{n} \leq v_{1} u_{1} v_{2} u_{2} \ldots v_{n} u_{n} v_{n+1}$ in the embedding order which by Lemma 4 yields that $v_{1}=v_{2}=\cdots=v_{n+1}=1$. But then $x_{1}=x_{2}=\cdots=x_{n+1}=e$, and $\left(\delta^{\prime}\right)$ holds.

(ii) $\Rightarrow$ (iii) trivially.

(iii) $\Rightarrow$ (i). By Theorem $2, M \cong \operatorname{Syn}(L)$ for an infix code $L$. Now let

$$
u_{1} u_{2} \cdots u_{n}, v_{1} u_{1} v_{2} u_{2} \cdots v_{n} u_{n} v_{n+1} \in L
$$

for some $u_{i}, v_{i} \in X^{*}$. Denoting $a_{i}=\left[u_{i}\right]$ and $x_{i}=\left[v_{i}\right]$ for all relevant indices $i$ and letting $c$ stand for $L$, we get $c=a_{1} a_{2} \cdots a_{n}=x_{1} a_{1} x_{2} a_{2} \cdots x_{n} a_{n} x_{n+1}$. By $\left(\gamma^{\prime \prime}\right)$, we have $x_{1}=x_{2}=\cdots=x_{n+1}=e$. In view of Lemma 2 , we conclude that $v_{1}=v_{2}=\cdots=v_{n+1}=1$ which finally shows that $L$ is a hypercode.

\section{THE THIRD THEOREM}

It deals with a special case of Theorem 2. A monoid $M$ with identity $e$ and zero 0 is said to be nil if for every $a \in M \backslash\{e\}$ there exists a positive integer $n$ such that $a^{n}=0$. Following [4], we call a monoid with zero nontrivial if it has at least three elements.

Theorem 3. A monoid $M$ is isomorphic to the syntactic monoid of an infix code $L$ over an alphabet $X$ with the property that for every $w \in X^{+}$there exists $n \geq 1$ such that $w^{n} \in W(L)$ if and only if $M$ is a subdirectly irreducible nontrivial nilmonoid.

Proof.

Necessity. Let $e$ be the identity of $M$. By Theorem $2(\alpha)$, we know that $M \backslash\{e\}$ is a subsemigroup of $M$. Since $W(L)$ is the zero of $\operatorname{Syn}(L)$, the 
hypothesis evidently implies that $M \backslash\{e\}$ is a nil semigroup and hence $M$ is a nilmonoid. By Theorem $2(\gamma), M$ has a disjunctive element $c$ different from $e$ and 0 and thus $M$ is nontrivial. In addition, $M \backslash\{e\}$ is a nilsemigroup having $c$ as a nonzero disjunctive element, which by [3, Theorem 4.5] implies that $M \backslash\{e\}$ is a subdirectly irreducible semigroup. Now [3, Theorem 3.6] yields that $M$ is a subdirectly irreducible monoid.

Sufficiency. By [5, Théorème I], subdirect irreducibility implies that $M$ has a core. Since $M$ is a nilmonoid, $M \backslash\{e\}$ is a nilsemigroup which evidently has a core, in fact equal to the core of $M \backslash\{e\}$ since $M \backslash\{e\}$ is a nonzero proper ideal of $M$. Now [3, Lemma 4.2] gives that $\operatorname{core}(M \backslash\{e\})=\mathscr{A}(M \backslash\{e\})$ and has exactly two elements. By [3, Theorem 3.6], subdirect irreducibility of $M$ implies that of $M \backslash\{e\}$. But then [3, Theorem 4.5] yields that $M \backslash\{e\}$ contains a nonzero disjunctive element. Now [3, Lemma 4.4] gives that 0 is a disjunctive element of $M \backslash\{e\}$. If $a \in M \backslash\{0, e\}$, then there exists $n \geq 1$ such that $a^{n} \neq 0$ and $a^{n+1}=0$, since $M \backslash\{e\}$ is a nilsemigroup, and thus $a a^{n} e=0$ and aee $=a \neq 0$. Therefore 0 is disjunctive in $M$.

We have proved that $M$ satisfies conditions $(\alpha),(\delta)$, and $(\epsilon)$ of Theorem 2 and thus $M \cong \operatorname{Syn}(L)$ for an infix code $L$ over an alphabet $X$. Let $w \in X^{+}$ and let $[w]$ be the corresponding element of $\operatorname{Syn}(L)$. By Lemma 2, $[w] \neq[1]$ and hence there exists $n \geq 1$ such that $[w]^{n}=0$ since $M$ is a nilmonoid. This means that $w^{n} \in W(L)$, as required.

A monoid $M$ with identity $e$ and zero 0 is said to be nilpotent if there exists a positive integer $n$ such that $a^{n}=0$ for all $a \in M \backslash\{e\}$.

Corollary 1. A monoid $M$ is isomorphic to the syntactic monoid of an infix code $L$ over an alphabet $X$ with the property that there exists $n \geq 1$ such that $w^{n} \in W(L)$ for every $w \in X^{+}$if and only if $M$ is a subdirectly irreducible nontrivial nilpotent monoid.

Proof.

Necessity. By Theorem 3, $M$ must be subdiretly irreducible and nontrivial. The additional hypothesis here evidently implies that for any $a \in M \backslash\{e\}$, we have $a^{n}=0$ and thus $M$ is a nilpotent monoid.

Sufficiency. By Theorem 3 , we have $M \cong \operatorname{Syn}(L)$ for some infix code $L$ over an alphabet $X$. Let $w \in X^{+}$. By Lemma 2, $[w] \neq[1]$ where these are $P_{L}$-classes. The hypothesis implies that $[w]^{n}=0$ and hence $w^{n} \in W(L)$.

Corollary 2 [2, Corollary 8 and Proposition 9]. A monoid $M$ is isomorphic to the syntactic monoid of a finite infix code over a finite alphabet if and only if $M$ is a finite subdirectly irreducible nontrivial nil monoid.

Following [4], we call a monoid strict if it satisfies condition $\left(\delta^{\prime}\right)$ in Corollary 1 to Theorem 2. 
Corollary 3 [4, Proposition 3]. A monoid $M$ is isomorphic to the syntactic monoid of a hypercode $L$ over a finite alphabet $X$ if and only if $M$ is a finite subdirectly irreducible nontrivial strict monoid.

Proof. This follows easily from Corollary 1 to Theorem 2, Corollary 2 to Theorem 3, [4, Corollary to Proposition 1] and the well-known statement that a hypercode over a finite alphabet must be finite.

\section{REFERENCES}

1. A. H. Clifford and G. B. Preston, The algebraic theory of semigroups. II, Amer. Math. Soc., Providence, RI, 1967.

2. H. Jürgensen and G. Thierrin, Infix codes, Proc. Fourth Hungarian Comp. Sci. Conf., Györ (1985), 25-29.

3. B. M. Schein, Homomorphisms of subdirect decompositions of semigroups, Pacific J. Math. 17 (1966), 529-547.

4. G. Thierrin, The syntactic monoid of a hypercode, Semigroup Forum 6 (1973), 227-231.

5. __ Sur la structure des demi-groupes, Alger Math. 3 (1956), 161-171.

Department of Mathematics, The University of Western Ontario, London, Ontario N6A 5B7 CANADA 